\title{
CHEMOTAXIS MODELS WITH A THRESHOLD CELL DENSITY
}

\author{
DARIUSZ WRZOSEK \\ Institute of Applied Mathematics and Mechanics, Warsaw University \\ Banacha 2, 02-097 Warszawa, Poland \\ E-mail: darekw@mimuw.edu.pl
}

\begin{abstract}
We consider a quasilinear parabolic system which has the structure of Patlak-KellerSegel model of chemotaxis and contains a class of models with degenerate diffusion. A cell population is described in terms of volume fraction or density. In the latter case, it is assumed that there is a threshold value which the density of cells cannot exceed. Existence and uniqueness of solutions to the corresponding initial-boundary value problem and existence of space inhomogeneous stationary solutions are discussed. In the 1D case a classification of stationary solutions for some model example is provided.
\end{abstract}

1. Introduction. We are dealing with a class of quasilinear systems of parabolic equations which are used to model the chemotactic motion of biological cells. Chemotaxis is understood here as a chemosensitive oriented movement of biological cells which may detect and response to some chemical (chemoattractant) secreted to their environment. It is assumed that the total flux of cells consists of diffusive and chemotactic parts. The classical model describing the aggregation phase of chemosensitive motion of cells was introduced by Patlak [11] and Keller and Segel [6]. Most of works on Patlak/Keller-Segel model were focused on the case when cell diffusion is Brownian and chemotactic sensitivity is a density-independent fixed constant. Recently new models which have nonlinearities in both chemotactic and diffusive parts have been introduced by Hillen and Painter $[2,10]$, and Byrne and Owen [3]. The latter is due to the multiphase modeling and the former takes into account a volume filling effect. For brevity we shall refer to (MP) and (VF) in the sequel. On the one hand, derivation and interpretation of both models are based on different approaches, but on the other one they turn out to share some common

2000 Mathematics Subject Classification: Primary 35K55, 35K65; Secondary 34B15, 34C25.

Key words and phrases: chemotaxis equations, degenerate diffusion, quasilinear parabolic equation, compactness method.

Supported by Polish KBN grant 2 P03A 03022.

The paper is in final form and no version of it will be published elsewhere. 
mathematical features. Both diffusive and chemotactic part contain here similar density dependent nonlinearities which lead in some cases to degenerate diffusion. Moreover, by different reasons in (MP) and (VF) a variable describing cell concentration is a priori bounded which precludes a blow-up of solution as a feature indicating the appearance of cells aggregate. Instead of it, a pattern formation mechanism was investigated for (VF) in $[2,10]$. Existence of flat-hump-shaped stationary solutions such that cells density attain the threshold value on some set with non-empty interior was recently proved in [8]. This type of solutions naturally refer to the formation of cells aggregate. The purpose of this work is just studying well-posedness and properties of stationary solutions to the class of chemotaxis models defined below. The present work extends methods used in an earlier related work [8]. In particular, the existence result proved here applies to (MP) model in some simplified version (MPs) which will be defined later.

More precisely, both models (VF) and (MPs) can be viewed in the following abstract form

$$
\begin{aligned}
& \partial_{t} u=\nabla \cdot(D(u) E(u, v) \nabla u-u \chi(u, v) \nabla v), \\
& \partial_{t} v=d \Delta v+g(u, v),
\end{aligned}
$$

in $(0, \infty) \times \Omega$ subjected to the no-flux boundary condition

$$
\langle D(u) E(u, v) \nabla u-u \chi(u, v) \nabla v \mid \nu\rangle=\langle\nabla v \mid \nu\rangle=0 \quad \text { on } \quad(0, \infty) \times \partial \Omega,
$$

and the initial condition

$$
(u, v)(0)=\left(u_{0}, v_{0}\right) \text { in } \Omega,
$$

where $\Omega$ is a bounded open subset of $\mathbb{R}^{N}$ with smooth boundary $\partial \Omega$ and $\nu$ is the outward unit normal vector on the boundary. Here, $u$ is interpreted in (VF) as the cell density and as a volume fraction in [3] where a two-phase model describing motion of cells in some fluid medium e.g water is introduced. Next, $v$ is a chemoattractant density and $D(u) E(u, v)$ denotes the density-dependent diffusion coefficient and $\chi(u, v)$ is usually named chemotactic sensitivity function. The function $g$ describes the rates of production and degradation of the chemoattractant $v$. Without loss of generality diffusion constant in (12) is normalized to 1 . We refer to a survey paper [5] for a detailed discussion and bibliography related to mathematical modelling of chemotaxis phenomenon. In particular, the classical Patlak-Keller-Segel-model of chemotaxis $[11,6]$ in the, so called, minimal version corresponds to the case where $D, E$ and $\chi$ are constants.

The volume filling model (VF) was formally derived in [10] from the level of a discrete space, continuous time, reinforced random walk on an equi-distant lattice. Let $u=u(x, t)$ denote the cell density at a position $x$ and time $t$ then $q(u(x, t))$ is introduced in [10] to determine the probability that a cell in a position $x$ and time $t$ finds space at its neighbouring location. Then $q=q(r)$ is a given non-negative and non-increasing function. Because of the volume filling effect in the tight packing state normalized here as $u \equiv 1$ we have $q(1)=0$. Consequently, it turns out that the chemotactic component of a total flux attains zero and the chemotactic movement stops when $u=1$. We then have

$$
D(r)=q(r)-r q^{\prime}(r), \quad E \equiv 1 \quad \text { and } \quad \chi(r, s)=q(r), \quad r \in[0,1] \times[0,+\infty) .
$$


If $q$ is assumed to be such that $D$ is bounded from below, e.g.

$$
q(r)=1-r,
$$

then (1)-(4) is a non-degenerate parabolic system. On the other hand, if we assume for example $q(r)=(1-r)^{2}$ then $D(r)=(1-r)(1+r)$ which leads to a degenerate parabolic system. Existence of a global in time and unique solution to to (VF) in the non-degenerate case was studied in $[2,10,13]$. In [14] it was proved that in the $\omega$-limit set of any trajectory to the corresponding dynamical system there are only stationary states provided $g$ is linear and (6) holds. Among them there are non-constant ones which never reach the threshold $u=1$.

In the degenerate case of (VF), model both diffusive and chemotactic parts of cells flux tend to 0 along with cells density tending to the threshold value. Existence and uniqueness of solutions to (VF) model in the degenerate case follows from [8] where also existence of stationary solutions which may reach the thereshold is shown in $1 \mathrm{D}$ case. We mention also [4] in which convergence of kinetic model for chemotaxis to the (VF) model is proved.

Recently Byrne and Owen have proposed a two-phase model describing cells moving through a fluid containing a diffusible chemoattractant. Now variable $u$ denotes a volume fraction of cells in a two component mixture that consists of cells and fluid (e.g. water) with volume fraction $w=1-u$. The derivation of the model is based on mass and momentum balance and some additional constitutive laws that describe interaction between cells, fluid and chemoattractant. Then changes in cells volume fraction are governed by (1) with the following substitution

$$
D(r)=\frac{r(1-r)^{2}}{k(r)}, \quad E=\Lambda(r, s)+r \partial_{r} \Lambda(r, s) \quad \text { and } \quad \chi(r, s)=\frac{r(1-r)^{2}}{k(r)} \partial_{s} \Lambda(r, s)
$$

for $(r, s) \in[0,1] \times[0,+\infty)$ where $k=k(r)$ is a drag function and $\Lambda(r, s)$ is a potential function which determines cells behavior. If one assumes diffusion to be a dominant factor causing the movement of chemoattractant molecules and neglect advection then fluctuation of chemoattractant in fluid may be described by an equation of the form (2). We call system (1) with (7) and (2) a simplified version (MPs) of full (MP) model which originally takes also into account the advection term in chemoattractant equation. The latter term leads, however, in general to inverse diffusion problem and even if one then restricts to a degenerate parabolic case it turns out to be non-triangular with gradient dependent reaction terms. This property leads to serious problems in proving well-posedness of global-in-time solutions.

Finally, we mention a hydrodynamical approach to chemotaxis equations proposed in [7]. Population of cells is treated there as a non-viscous fluid with density $u$ and velocity $\mathbf{v}$ which satisfies Euler equation

$$
\begin{aligned}
\partial_{t}+\nabla \cdot(u \mathbf{v}) & =0, \\
u\left(\partial_{t} \mathbf{v}+\mathbf{v} \cdot \nabla \mathbf{v}\right) & =-\nabla p+F(u, v, \mathbf{v})
\end{aligned}
$$

with the density-dependent pressure $p=p(u)$ and the force

$$
F=\mu \nabla v-\beta(u) \mathbf{v}
$$


composed from chemotactic part oriented towards gradient of chemoattractant $v$ and resistive force which is due to all factors moderating cell movement in particular kinematic friction. We note that originally $\beta$ is taken as a constant friction coefficient in [7]. Then, assuming that inertial force is negligible for the description of cells movement we obtain following [7]

$$
\mu \nabla v-\nabla p(u)-\beta(u) \mathbf{v}=0 .
$$

Hence, using (8) we arrive at

$$
\partial_{t} u=\nabla \cdot\left(\frac{u p^{\prime}(u)}{\beta(u)} \nabla u-\frac{\mu u}{\beta(u)} \nabla v\right) .
$$

It is then easy to check that, setting in the above equation $q(r)=\beta(r)^{-1}$ and $p(r)=$ $\ln (r \beta(r))$, we obtain (5) in (VF) model. It shows that derivations of chemotaxis equations based on entirely different approaches lead to similar structures which are linked at least on a formal level.

We are now in a position to list the assumptions that we impose on the data. The functions $D \in \mathcal{C}^{2}(\mathbb{R}), \chi \in \mathcal{C}^{2}(\mathbb{R})$ satisfy

$$
\begin{array}{cl}
D(0) \geq 0, \quad D(1) \geq 0 \quad \text { and } \quad D(r)>0 & \text { for } \quad r \in(0,1), \\
\chi(1, s)=0, \quad \chi(r, s)>0 & \text { for } \quad(r, s) \in(0,1) \times[0,+\infty) .
\end{array}
$$

Notice that we are mainly interested in the degenerate case when

$$
D(0)=0 \text { and } / \text { or } D(1)=0 .
$$

There is $e_{0}>0$ such that

$$
E(r, s) \geq e_{0} \quad \text { for all }(r, s) \in(0,1) \times[0,+\infty),
$$

and that the reaction term $g \in \mathcal{C}^{2}\left(\mathbb{R}^{2}\right)$ satisfies the (one-sided) growth condition

$$
g(r, 0) \geq 0 \quad \text { and } \quad \partial_{s} g(r, s) \leq \kappa \quad \text { for } \quad(r, s) \in[0,1] \times[0, \infty),
$$

for some $\kappa>0$.

As for the initial data, we assume that

$$
\left(u_{0}, v_{0}\right) \in L^{\infty}\left(\Omega ; \mathbb{R}^{2}\right) \text { with } 0 \leq u_{0} \leq 1 \text { and } 0 \leq v_{0} \text { a.e. in } \Omega .
$$

At last, we note that assumptions (11), (12) and (14) comprise most of examples studied in [10] and [3]. In particular, we may have $k(r)=$ const, $k(r)=(1-r), k(r)=$ $r(1-r)$ and $\Lambda(r, s)=\alpha+\gamma \exp \left\{-\frac{\theta s}{1-r}\right\}$ (cf. [3]).

As it was mentioned before, it is an interesting question whether variable $u$ may attain the threshold density since the set $\{x \in \Omega: u(t, x)=1\}$ indicates the set of points where cells are tightly packed or for the case of (MPs) model the set of points where solely pure phase occurs. This problem was studied in [8] concerning stationary solutions. It turns out that a stationary solution $(u, v)$ to (1)-(2) under assumption (40) and $D(1)=0, D(0)>0, \chi(r, s)=\chi(r)$ satisfies for some $\lambda \in \mathbb{R}$ the following relation

$$
j(u(x))=v(x)+\lambda \quad \text { if } \quad u \in(0,1),
$$

where $j(r):=\int_{1 / 2}^{r} \frac{D(s)}{s \chi(s)} d s$ for $r \in(0,1)$. Two cases have been distinguished in [8]: nondegenerate case when $D / s \chi(s) \notin L^{1}(1 / 2,1)$ and degenerate one if $D / s \chi(s) \in L^{1}(1 / 2,1)$. 
It was shown that in the non-degenerate case we have $0<u<1$ and the threshold is never reached in this case. On the contrary, in the degenerate case the existence of a broad class of stationary solutions in $1 \mathrm{D}$ which takes value 1 on some subinterval of $[0, l]$ was proved. Such a flat-hump-shaped solution exists provided the length $l$ of the interval is big enough. A degenerate case under simplifying assumption $D(r)=r \chi(r), j$ is studied here. It is proved that $j$ is an affine function with $j(0)<+\infty, j(1)<+\infty$ and then non-homogeneous stationary solutions exist in arbitrary space dimension provided the domain of definition has some specific properties related to eigenvalue problem for the Laplace operator with the homogeneous Neumann boundary condition (Theorem 6). In $1 \mathrm{D}$ case several types of stationary solutions including flat-hump shaped ones are distinguished. In Theorem 8 a classification of stationary states for this case is provided.

The same concept of a weak solution to (1)-(4) as in [8] is used in this paper. First we define

$$
\mathcal{D}(r):=\int_{0}^{r} D(s) d s, \quad r \in[0,1] .
$$

Definition 1. Let $\left(u_{0}, v_{0}\right) \in L^{\infty}\left(\Omega ; \mathbb{R}^{2}\right)$ be such that (16) holds true. A weak solution to (1)-(4) is a couple $(u, v)$ of functions such that, for each $T>0$,

$$
\begin{gathered}
0 \leq u(t, x) \leq 1, \quad 0 \leq v(t, x) \quad \text { a.e. in } \quad(0, T) \times \Omega, \\
u \in L^{\infty}((0, T) \times \Omega) \cap \mathcal{C}_{w}\left([0, T] ; L^{2}(\Omega)\right), \quad \mathcal{D}(u) \in L^{2}\left(0, T ; H^{1}(\Omega)\right), \quad u(0)=u_{0}, \\
v \in L^{\infty}((0, T) \times \Omega) \cap H^{1}\left(0, T ; L^{2}(\Omega)\right) \cap L^{2}\left(0, T ; H^{2}(\Omega)\right), \quad v(0)=v_{0},
\end{gathered}
$$

and $(u, v)$ satisfy

$$
\begin{aligned}
\int_{0}^{T}\left\langle\partial_{t} u, \psi\right\rangle d t & +\int_{0}^{T} \int_{\Omega}(E(u, v) \nabla \mathcal{D}(u)-u \chi(u, v) \nabla v) \nabla \psi(t, x) d x d t=0 \\
\partial_{t} v & =\Delta v+g(u, v) \quad \text { a.e. in } \quad(0, T) \times \Omega \\
\partial_{\nu} v & =0 \quad \text { a.e. on }(0, T) \times \partial \Omega
\end{aligned}
$$

for each $t \in[0, T]$ and $\psi \in L^{2}\left(0, T ; H^{1}(\Omega)\right)$. Here, $\langle.,$.$\rangle denotes the duality pairing between$ $H^{1}(\Omega)$ and $H^{1}(\Omega)^{\prime}$.

Here, $\mathcal{C}_{w}\left([0, T] ; L^{2}(\Omega)\right)$ denotes the set of functions from $[0, T]$ in $L^{2}(\Omega)$ which are continuous for the weak topology of $L^{2}(\Omega)$. It is worth to mention that well-posedness to system (1)-(4) was studied in [8] under similar assumptions with the only difference $E \equiv$ const and $D(0)>0$. Then, existence of weak solutions was proved in the aforementioned paper (Theorem 2) and also uniqueness was shown by the duality method provided $D$ and $\chi$ satisfy some additional condition. It turns out that the method used to prove existence of weak solution can be extended to our case. However, the uniqueness problem requires, in general, a new method since that from [8] can be adopted here if $E(r, s)$ does not depend on $s$, and $\chi$ is factorized as $\chi(r, s)=\chi_{1}(r) \chi_{2}(s)$ (see Proposition 3$)$. This condition is usually satisfied in most models taking into account a chemoattractant-dependent chemotactic sensitivity function $\chi$. 
2. Existence and uniqueness. We shall prove

Theorem 2 (Existence). Consider $\left(u_{0}, v_{0}\right) \in L^{\infty}\left(\Omega ; \mathbb{R}^{2}\right)$ such that (16) holds true. Under the assumptions (11), (12), (14), (15) on $D, \chi$ and $g$, there exists a weak solution $(u, v)$ to (1)-(4) in the sense of Definition 1 such that

$$
\int_{\Omega} u(t, x) d x=\int_{\Omega} u_{0}(x) d x \text { for each } t \geq 0 .
$$

Since the proof this theorem requires only slight modifications with respect to the proof of [8, Theorem 2.], we shall give only a sketch indicating differences. We introduce first a parameter which determines a sequence of solutions to non-degenerate regularized problems for which the well-posedness is deduced from the abstract theory developed by Amann [1] and then using compactness argument one can extract a subsequence which is convergent in an appropriate sense to the weak solution.

Proof. In the non-degenerate case $D(0)>0, D(1)>0$ existence and uniqueness of classical solutions follows from Amann's theory of quasilinear parabolic equations applied below to solve the regularized problem.

Step 1. Regularization. For $\varepsilon=\frac{1}{m}, m>1$ and $r \in \mathbb{R}$, we put $D^{\varepsilon}(r):=D(r)+\varepsilon$. To aproximate the initial data we take $\left(u_{0}^{\varepsilon}, v_{0}^{\varepsilon}\right) \in W^{1, N+1}\left(\Omega ; \mathbb{R}^{2}\right)$ such that $(16)$ and

$$
\left\|v_{0}^{\varepsilon}\right\|_{L^{\infty}} \leq 2\left\|v_{0}\right\|_{L^{\infty}} \quad \text { and } \quad\left\|u_{0}^{\varepsilon}-u_{0}\right\|_{L^{2}}+\left\|v_{0}^{\varepsilon}-v_{0}\right\|_{L^{2}} \leq \varepsilon .
$$

Next we consider the initial-boundary value problem (1)-(4) with $D^{\varepsilon}$ instead of $D$ and $\left(u_{0}^{\varepsilon}, v_{0}^{\varepsilon}\right)$ instead of $\left(u_{0}, v_{0}\right)$. The elliptic part of (1)-(4) is upper triangular in separated divergence form. Therefore, the problem is normally elliptic by [1, Section 4]. We are then in a position to apply [1, Theorems $14.4 \& 14.6]$ to conclude that (1)-(4) has a unique maximal classical solution

$$
\left(u^{\varepsilon}, v^{\varepsilon}\right) \in \mathcal{C}\left(\left[0, T_{\star}\right) \times \bar{\Omega} ; \mathbb{R}^{2}\right) \cap \mathcal{C}^{1,2}\left(\left(0, T_{\star}\right) \times \bar{\Omega} ; \mathbb{R}^{2}\right),
$$

where $T_{\star} \in(0,+\infty]$. Next $(1)$ and the fact that $g(\cdot, 0) \geq 0$ and [1, Theorem 15.1] ensure that $u^{\varepsilon}(t, x) \geq 0$ and $v^{\varepsilon}(t, x) \geq 0$ for $(t, x) \in\left[0, T_{\star}\right) \times \bar{\Omega}$. We next observe that $\tilde{z}:=$ $\left(v^{\varepsilon}, 1-u^{\varepsilon}\right)$ is a solution to an initial-boundary value problem with a similar structure to (1)-(2). Since $\chi(1)=0$, a further application of [1, Theorem 15.1] leads to the conclusion that $1-u^{\varepsilon} \geq 0$. Finally we obtain for $t \in[0, T]$

$$
0 \leq u^{\varepsilon}(t, x) \leq 1 \quad \text { and } \quad 0 \leq v^{\varepsilon}(t, x)
$$

for $(t, x) \in\left[0, T_{\star}\right) \times \bar{\Omega}$. Also, integrating $(1)$ over $(0, t) \times \Omega$ implies the mass conservation (28). We finally infer from (2), (15) and (26) that

$$
\partial_{t} v^{\varepsilon}-\Delta v^{\varepsilon} \leq g\left(u^{\varepsilon}, 0\right)+\kappa v^{\varepsilon} \leq C\left(1+v^{\varepsilon}\right)
$$

with $C:=\|g(., 0)\|_{L^{\infty}(0,1)}+\kappa$, from which we conclude that

$$
v^{\varepsilon}(t, x) \leq\left(1+\left\|v_{0}\right\|_{L^{\infty}}\right) e^{C t}, \quad(t, x) \in\left[0, T_{\star}\right) \times \bar{\Omega} .
$$

Since the elliptic boundary value problem has a triangular structure, $L^{\infty}$-bound is sufficient to conclude ([1, Theorem 15.5]) that the maximal solution is in fact global in time: 
$T_{\star}=+\infty$, and thanks to no-flux boundary condition we have

$$
\int_{\Omega} u^{\varepsilon}(t, x) d x=\int_{\Omega} u_{0}^{\varepsilon}(x) d x \text { for each } t \geq 0 .
$$

In the following we denote by $C_{i}, i \geq 1$, any positive constant which does not depend on $\varepsilon \in(0,1)$. The dependence of the $C_{i}$ 's upon additional parameters will be indicated explicitly.

Consequently, the right-hand side of (2) is bounded on finite time intervals and classical parabolic estimates warrant that, for each $T>0$ and $p \in(1, \infty)$, there is a positive constant $C_{2}(p, T)$ such that

$$
\left\|v^{\varepsilon}\right\|_{L^{p}\left(0, T ; W^{2, p}(\Omega)\right)}+\left\|\partial_{t} v^{\varepsilon}\right\|_{L^{p}((0, T) \times \Omega)} \leq C_{1}(p, T) .
$$

Step 2. Compactness. We next introduce the functions $\mathcal{D}^{\varepsilon}$ and $\tilde{\mathcal{D}}^{\varepsilon}$ defined by

$$
\frac{d^{2} \tilde{\mathcal{D}}^{\varepsilon}}{d r^{2}}=\frac{d \mathcal{D}^{\varepsilon}}{d r}=D^{\varepsilon} \quad \text { and } \quad \tilde{\mathcal{D}}^{\varepsilon}(0)=\mathcal{D}^{\varepsilon}(0)=0 .
$$

We multiply (1) by $\mathcal{D}^{\varepsilon}\left(u^{\varepsilon}\right)$, integrate over $\Omega$ and using (14) we obtain

$$
\begin{aligned}
\frac{d}{d t} \int_{\Omega} \tilde{\mathcal{D}}^{\varepsilon}\left(u^{\varepsilon}\right) d x & =-\int_{\Omega}\left(E\left(u^{\varepsilon}, v^{\varepsilon}\right)\left|\nabla \mathcal{D}^{\varepsilon}\left(u^{\varepsilon}\right)\right|^{2}-u^{\varepsilon} \chi\left(u^{\varepsilon}, v^{\varepsilon}\right) \nabla v^{\varepsilon} \nabla \mathcal{D}^{\varepsilon}\left(u^{\varepsilon}\right)\right) d x \\
& \leq-e_{0}\left\|\nabla \mathcal{D}^{\varepsilon}\left(u^{\varepsilon}\right)\right\|_{L^{2}}^{2}+\left\|\chi\left(u^{\varepsilon}, v^{\varepsilon}\right)\right\|_{L^{\infty}(\Omega)}\left\|\nabla \mathcal{D}^{\varepsilon}\left(u^{\varepsilon}\right)\right\|_{L^{2}}\left\|\nabla v^{\varepsilon}\right\|_{L^{2}} \\
& \leq-\frac{e_{0}}{2}\left\|\nabla \mathcal{D}^{\varepsilon}\left(u^{\varepsilon}\right)\right\|_{L^{2}}^{2}+\frac{\left\|\chi\left(u^{\varepsilon}, v^{\varepsilon}\right)\right\|_{L^{\infty}(\Omega)}^{2}}{2}\left\|\nabla v^{\varepsilon}\right\|_{L^{2}}^{2},
\end{aligned}
$$

whence in view of (29 it follows that

$$
\int_{0}^{T} \int_{\Omega}\left|\nabla \mathcal{D}^{\varepsilon}\left(u^{\varepsilon}\right)\right|^{2} d x d t \leq C_{2}(T)
$$

and also

$$
\int_{0}^{T} \int_{\Omega}\left|\nabla \mathcal{D}\left(u^{\varepsilon}\right)\right|^{2} d x d t \leq C_{2}(T)
$$

since $d \mathcal{D}^{\varepsilon} / d r \geq D \geq 0$. Next from (1), (29) and (30) we deduce that

$$
\int_{0}^{T}\left\|\partial_{t} u^{\varepsilon}\right\|_{H^{1}(\Omega)^{\prime}}^{2} d t \leq C_{4}(T) .
$$

Since the $L^{2}$ estimate on $\nabla u^{\varepsilon}$ is not available here, the compactness argument is applied to an auxiliary sequence $P\left(u^{\varepsilon}\right)$ where $P \in \mathcal{C}^{2}(\mathbb{R})$ and $P(0)=0$ with $d P / d r:=D^{2}$. Then, proceeding in the same way as in [8], one can show using (1), (14), (29), (32), (31) that

$$
\left(P\left(u^{\varepsilon}\right)\right) \text { is bounded in }\left\{w \in L^{2}\left(0, T ; H^{1}(\Omega)\right): \partial_{t} w \in L^{1}\left(0, T ; W^{1, N+1}(\Omega)^{\prime}\right)\right\}
$$

for each $T>0$. Then applying a compactness result [12, Corollary 4] we conclude that $\left(P\left(u^{\varepsilon}\right)\right)$ is relatively compact in $L^{2}((0, T) \times \Omega)$ for each $T>0$. It follows from (1) that $P$ is an increasing function and therefore for a subsequence $\left(u^{\varepsilon}\right)$ is almost everywhere convergent. This property combined with the bound (26) yields the relative compactness of $\left(u^{\varepsilon}\right)$ in $L^{p}((0, T) \times \Omega)$ for each $p \in[1, \infty)$ and $T>0$. Also, $(26),(32)$ and a classical compactness result $[9$, Théorème 1.12 .1$]$ ensure that $\left(u^{\varepsilon}\right)$ is relatively compact in $\mathcal{C}\left([0, T] ; H^{1}(\Omega)^{\prime}\right)$ 
for each $T>0$. It follows that there are functions $(u, v) \in L^{\infty}\left((0, T) \times \Omega ; \mathbb{R}^{2}\right)$ and a subsequence of $\left(u^{\varepsilon}, v^{\varepsilon}\right)$ (not relabeled) such that

$$
\left(u^{\varepsilon}, v^{\varepsilon}\right) \rightarrow(u, v) \quad \text { in } \quad L^{p}\left((0, T) \times \Omega ; \mathbb{R}^{2}\right),
$$

and

$$
\left(u^{\varepsilon}, v^{\varepsilon}\right) \rightarrow(u, v) \quad \text { in } \quad \mathcal{C}\left([0, T] ; H^{1}(\Omega)^{\prime}\right) \times \mathcal{C}\left([0, T] ; L^{p}(\Omega)\right),
$$

for each $p \in[1, \infty)$ and $T>0$. Furthermore, $(31)$ ensures that $\mathcal{D}(u) \in L^{2}\left(0, T ; H^{1}(\Omega)\right)$ for each $T>0$. Since $D^{\varepsilon}\left(u^{\varepsilon}\right)$ is weakly convergent in $L^{2}\left(0, T ; H^{1}(\Omega)\right)$ for each $T>0$ and by $(14),(26),(27),(35)$ we have for $p \in[0,+\infty)$

$$
E\left(u^{\varepsilon}, v^{\varepsilon}\right) \rightarrow E(u, v) \quad \text { in } \quad L^{p}\left((0, T) \times \Omega ; \mathbb{R}^{2}\right),
$$

It follows, using standard arguments, that for any $\psi \in L^{2}\left(0, T ; H^{1}(\Omega)\right)$

$$
\int_{0}^{T} \int_{\Omega} E\left(u^{\varepsilon}, v^{\varepsilon}\right) \nabla \mathcal{D}^{\varepsilon}\left(u^{\varepsilon}\right) \nabla \psi(t, x) d x d t \rightarrow \int_{0}^{T} \int_{\Omega} E(u, v) \nabla \mathcal{D}(u) \nabla \psi(t, x) d x d t,
$$

Similarly, using (29) one can pass to the limit in the chemotactic part and thanks to (35) we infer that (21) is satisfied. Then, making use of (29) and (34) we pass to the limit in the weak formulation to (22), and then using the classical regularity theory of parabolic equations we infer that (22) is satisfied in the strong sense. Hence, in view of $(35),(u, v)$ is a solution to (1)-(4) in the sense of Definition 1.

Uniqueness of weak solution can be proved using duality method following lines of proof of $[8$, Theorem 2] provided

$$
E \equiv \text { const and } \chi(r, s)=\chi_{1}(r) \chi_{2}(s),
$$

where $\chi_{1}, \chi_{2} \in \mathcal{C}^{2}$ and $\chi$ satisfies (12). We next assume that there are $C_{0}>0, C_{1}>0$ and functions $g_{1} \in \mathcal{C}^{2}(\mathbb{R})$ and $g_{2} \in \mathcal{C}^{2}(\mathbb{R})$ such that $g_{1}(0) \geq 0, g_{2}(0) \geq 0$ and

$$
g(r, s)=g_{1}(s)+r g_{2}(s) \text { and } \max \left\{g_{1}^{\prime}(s), g_{2}^{\prime}(s)\right\} \leq C_{1},
$$

for $(r, s) \in[0,1] \times[0, \infty)$.

Proposition 3 (Uniqueness). If (36)-(37) are satisfied and additionally

$$
\left(r \chi_{1}(r)-s \chi_{1}(s)\right)^{2} \leq C_{0}(r-s)(\mathcal{D}(r)-\mathcal{D}(s)),
$$

then the weak solution is unique.

3. Stationary states. In this section, we shall study stationary solutions to (1)-(3) in a particular case when (11)-(12) hold and

$$
D(r)=r h(r), \text { and } \chi(r)=\chi_{0} h(r),
$$

where $h$ is a sufficiently regular function satisfying (11)-(12) and $\chi_{0}$ is a positive constant that determines the strength of the chemotactic force. Moreover, we assume

$$
E \equiv 1, \quad g(r, s):=\gamma r-\beta s
$$


for some $\beta>0$ and $\gamma>0$. The case of linear $g$ was studied in most of works on chemotaxis equation. The following system is an example:

$$
\begin{aligned}
\nabla \cdot\left(u(1-u) \nabla u-\chi_{0} u(1-u) \nabla v\right) & =0, \\
\Delta u+\gamma u-\beta v & =0 .
\end{aligned}
$$

The same concept of stationary solution as in [8] is used here.

Definition 4. A stationary solution to (1)-(3) is a couple $(u, v)$ of functions in $L^{\infty}\left(\Omega ; \mathbb{R}^{2}\right)$ such that $\mathcal{D}(u) \in H^{1}(\Omega), v \in H^{2}(\Omega)$,

$$
\begin{aligned}
& (u(x), v(x)) \in[0,1] \times[0,+\infty) \quad \text { a.e. in } \Omega, \\
& \nabla \mathcal{D}(u)-u \chi(u) \nabla v=0 \quad \text { a.e. in } \Omega, \\
& -\Delta v+\beta v=\gamma u \quad \text { a.e. in } \Omega, \\
& \partial_{\nu} v=0 \quad \text { a.e. on } \partial \Omega .
\end{aligned}
$$

If $M \in[0,|\Omega|]$ then a stationary solution to (1)-(3) with mass $(M, \gamma M / \beta)$ satisfies

$$
\int_{\Omega} u(x) d x=\frac{\beta}{\gamma} \int_{\Omega} v(x) d x=M .
$$

Of course, there is a one-parameter family of space-homogeneous solutions

$$
C_{\varrho}=\left\{(\tilde{u}, \tilde{v}): \tilde{u} \equiv \frac{\gamma}{\beta} \varrho, \tilde{v} \equiv \varrho\right\}, \quad\{\varrho \in[0, \gamma / \beta]\},
$$

and there arises a question whether non-homogeneous stationary solutions exist. Following [8] we introduce the auxiliary function

$$
j(r):=\int_{1 / 2}^{r} \frac{D(s)}{h(s)} d s \quad \text { for } r \in(0,1) .
$$

It was shown in [8] that in the case when

$$
D(0)>1, \quad D(1)=0 \text { and } \chi(1)=0,
$$

$j$ is an increasing function from $(0,1)$ onto $\left(-\infty, j_{1}\right)$, where

$$
j_{1}:=\int_{1 / 2}^{1} \frac{D(s)}{h(s)} d s \in(0,+\infty],
$$

and for any stationary solution $(u, v)$ with mass $(M, \gamma M / \beta), M \in(0,|\Omega|)$, the set

$$
\Omega_{u}:=\{x \in \Omega, u(x) \in[0,1)\}
$$

is a non-empty open subset of $\Omega$. Moreover, $j(u)-v$ is constant on each connected component of $\Omega_{u}$ and $u \in \mathcal{C}(\bar{\Omega}) \cap \mathcal{C}^{1}\left(\Omega_{u}\right)$. In addition, $u(x)>0$ for $x \in \bar{\Omega}$ and by comparison principle

$$
v(x) \in\left[0, \frac{\gamma}{\beta}\right], \quad x \in \bar{\Omega} .
$$

If we assume (39)-(40) $j$ is an affine function

$$
j(r)=\frac{1}{\chi_{0}}\left(r-\frac{1}{2}\right),
$$


and in contrast to the previously studied case $j$ satisfies both $j(0)=-\frac{1}{2 \chi_{0}}$ and $j(1)=\frac{1}{2 \chi_{0}}$, so this is a doubly degenerate case in the terminology of [8]. We recall that in the case (46) flat-hump-shaped solutions ( $u=1$ on some set with non-empty interior) may appear only in the degenerate case i.e. $j(1)<+\infty$.

Similar arguments as in [8, Proposition 5] lead to

Proposition 5. Under assumptions (39)-(41) for a stationary solution $(u, v)$ with mass $(M, \gamma M / \beta), M \in(0,|\Omega|)$, the set $\Omega_{u}^{\prime}:=\{x \in \Omega, u(x) \in(0,1)\}$ is a non-empty open subset of $\Omega$. Moreover, in view of (48) on each connected component of $\Omega_{u}^{\prime}$ we have for some $\lambda \in \mathbb{R}$

$$
u=\chi_{0}(v-\lambda)+\frac{1}{2}
$$

and $u \in \mathcal{C}(\bar{\Omega}) \cap \mathcal{C}^{1}\left(\Omega_{u}^{\prime}\right)$. Thus

$$
\Omega \backslash \Omega_{u}^{\prime}=\Omega_{1} \cup \Omega_{0},
$$

where

$$
\Omega_{0}=\{x \in \Omega: u(x)=0\} \text { and } \Omega_{1}=\{x \in \Omega: u(x)=1\} .
$$

Moreover, the set of stationary solutions has the following symmetry: if a couple $(u, v)$ is a stationary solution then $(w, z)$ is also a stationary solution with $w=1-u$ and $z=\frac{\gamma}{\beta}-v$.

The last statement can be checked by a direct computation.

Requirements $0<u(x)<1$ for $x \in \bar{\Omega}$ lead to the following restrictions on $v$ and $\lambda$

$$
\lambda-\frac{1}{2 \chi_{0}}:=v_{0}<v(x)<v_{1}:=\lambda+\frac{1}{2 \chi_{0}},
$$

and (47) holds. Combining (47) and (50) we obtain

$$
\lambda \in \Lambda:=\left[\frac{1}{2 \chi_{0}}, \frac{\gamma}{\beta}-\frac{1}{2 \chi_{0}}\right] .
$$

It follows that $\Lambda \neq \emptyset$ if

$$
\beta \leq \gamma \chi_{0}
$$

Using (49) and (50) we may introduce

$$
u(x)=F_{\lambda}(v(x)) \text { for } x \in \bar{\Omega},
$$

where

$$
F_{\lambda}(y)=\left\{\begin{array}{cl}
\chi_{0}(y-\lambda)+\frac{1}{2} & \text { for } v_{0}<y<v_{1} \\
1 & \text { for } y>v_{1} \\
0 & \text { for } 0<y<v_{0} .
\end{array}\right.
$$

Next, (43) and (52) yield that

$$
-\Delta v=\gamma F_{\lambda}(v)-\beta v \text { in } \Omega
$$

with the no-flux boundary condition on $\partial \Omega$. Observe that the continuity of $v$ and (54) imply that for $i=0,1$, we have

$$
\begin{aligned}
-\Delta v & =\gamma i-\beta v \quad \text { in } \Omega_{i}, \\
v & =v_{i} \text { on } \partial \Omega_{i} .
\end{aligned}
$$


Denote the mean value of $f$ by $\bar{f}=\frac{1}{|\Omega|} \int_{\Omega} f(x) d x$ and

$$
\omega^{2}:=\gamma \chi_{0}-\beta \text {. }
$$

Let $S=S(\Omega)$ denote the set of eigenvalues corresponding to the following eigenvalue problem

$$
\begin{aligned}
\Delta w=-\mu^{2} w & \text { in } \quad \Omega, \\
\partial_{\nu} w=0 \quad \text { on } & \partial \Omega .
\end{aligned}
$$

THEOREM 6. If

$$
\omega^{2} \in S(\Omega) \backslash\{0\}
$$

then for any $\varrho \in\left(0, \frac{\gamma}{\beta}\right)$ there exists a space non-homogeneous stationary solution $(u, v)$ such that $\bar{v}=\varrho, \bar{u}=\frac{\beta}{\gamma} \varrho$ and $0<u(x)<1,0<v(x)$ for $x \in \Omega$ and

$$
v=A w_{\omega}+\bar{v}, \quad u=\chi_{0}(v-\lambda)+1 / 2,
$$

where $w_{\omega}$ is an arbitrary but fixed eigenfunction corresponding to (57)-(58) with $\mu=\omega$ and

$$
\lambda=\frac{\omega^{2} \bar{v}}{\gamma \chi_{0}}+\frac{1}{2 \chi_{0}}
$$

and $A>0$ satisfies

$$
0<A<\min \left\{\frac{\beta \bar{v}}{\gamma \chi_{0}\left|\min _{x \in \Omega}\left\{w_{\omega}\right\}\right|}, \frac{\gamma-\beta \bar{v}}{\gamma \chi_{0} \max _{x \in \Omega}\left\{w_{\omega}\right\}}\right\} .
$$

Proof. We fix $\varrho \in\left(0, \frac{\gamma}{\beta}\right)$. In view of (49) we consider the following boundary value problem

$$
\begin{aligned}
\Delta v & =-\gamma\left(\chi_{0} v-\chi_{0} \lambda+1 / 2\right)+\beta v=-\omega^{2} v+\gamma\left(\chi_{0} \lambda-1 / 2\right) \text { a.e. in } \Omega, \\
\partial_{\nu} v & =0 \text { a.e. on } \partial \Omega,
\end{aligned}
$$

which has a solution

$$
v=A w_{\omega}+\frac{\gamma\left(\chi_{0} \lambda-1 / 2\right)}{\omega^{2}},
$$

where $w_{\omega}$ is a fixed eigenfunction related to (57)-(58). Since $\int_{\Omega} w_{\omega}(x) d x=0$, we easily check that $\varrho=\bar{v}$, and then by $(50)$

$$
v_{0}=\frac{w^{2}}{\gamma \chi_{0}} \bar{v}, \quad v_{1}=\frac{1}{\chi_{0}}-\frac{w^{2}}{\gamma \chi_{0}} \bar{v} .
$$

Since we used (49) in (62), the requirement $v_{0}<v(x)<v_{1}$ for $x \in \Omega$ yields bounds (61) on $A$. This completes the proof.

COROllary 7. 1. Non-homogeneous stationary solutions $(u, v)$ satisfying condition $0<$ $u<1$ may exist only on special domains $\Omega$ for which the eigenvalue problem for the Laplace operator with the Neumann homogeneous boundary condition satisfies (59).

2. There is a continum of nonhomogeneous stationary solutions corresponding to a given mass.

From now on, we restrict ourselves to the one-dimensional case, $\Omega=I=(0, l)$, and in view of $(54)$ we seek a solution $(u, v): I \rightarrow[0,1] \times \mathbb{R}_{+}$to the following boundary-value 
problem

$$
\begin{aligned}
v^{\prime \prime} & =\Phi_{\lambda}(v) \quad \text { in }(0, l), \quad v^{\prime}(0)=v^{\prime}(l)=0, \\
\Phi_{\lambda}(v) & =-\gamma F_{\lambda}(v)+\beta v,
\end{aligned}
$$

where $F_{\lambda}$ satisfies (53) and $u$ is given by (52). We denote

$$
I_{0}=\{x \in I: u(x)=0\}, \quad I_{1}=\{x \in I: u(x)=1\} .
$$

We define the following sets $K, K_{i}, K_{i}^{\prime}, L^{\prime}, L$ of vector-valued functions $(w, z): I \rightarrow$ $[0, l] \times \mathbb{R}_{+}$with the following symmetry property $(w(x), z(x))=(w(l-x), z(l-x))$ for $x \in l$ and

$$
\begin{aligned}
& (w, z) \in K \text { iff } I_{0}=I_{1}=\emptyset, l=2 \pi / \omega, i=0,1, \\
& (w, z) \in K_{i} \text { iff } I_{i} \neq \emptyset \text { is an interval, } I_{i+1(\bmod 2)}=\emptyset, l>2 \pi / \omega, i=0,1, \\
& (w, z) \in K_{i}^{\prime} \text { iff } \operatorname{card}\left\{I_{i}\right\}=1 \text { and } I_{i+1(\bmod 2)}=\emptyset, l=2 \pi / \omega, \\
& (w, z) \in L^{\prime} \text { iff } \operatorname{card}\left\{I_{0}\right\}=\operatorname{card}\left\{I_{1}\right\}=2, l=2 \pi / \omega, \\
& (w, z) \in L \text { iff } \operatorname{card}\left\{I_{0}\right\}>1, \operatorname{card}\left\{I_{1}\right\}>1, l>2 \pi / \omega .
\end{aligned}
$$

THEOREM 8. If $\gamma \chi_{0}-\beta>0$ then there are space non-homogeneous stationary solutions $(u, v)$ to (1)-(3) in the one-dimensional case.

1. If $\lambda=\frac{\gamma}{2 \beta}$ then $K \cup L \cup L^{\prime} \neq \emptyset$.

2. If $\lambda<\frac{\gamma}{2 \beta}$ then $K \cup K_{0} \cup K_{0}^{\prime} \neq \emptyset$, if $u \neq 0$ then $u \neq 1$.

3. If $\lambda>\frac{\gamma}{2 \beta}$ then $K \cup K_{1} \cup K_{1}^{\prime} \neq \emptyset$, if $u \neq 1$ then $u \neq 0$.

If $\gamma \neq 2 \beta$ and $\bar{u}<1 / 2$ then any solution to (65) belongs to $K \cup K_{0} \cup K_{0}^{\prime}$. If $(u, v)$ is a stationary solution, then

$$
v \in \mathcal{C}^{2}([0, l]), \quad u \in \mathcal{C}([0, l]), \text { and } \mathcal{D}(u) \in \mathcal{C}^{1}([0, l]) .
$$

Proof. Since $\gamma \chi_{0}-\beta>0$ for $\lambda \in \Lambda$ there are 3 points $P_{0}=(0,0), P_{1}=\left(v_{e}, \frac{\beta}{\gamma} v_{e}\right), P_{2}=$ $\left(\frac{\gamma}{\beta}, 1\right)$ at which the graph of function $z=F_{\lambda}(y)$ meets the line $z=\frac{\beta}{\gamma} y ; 0<v_{0}<v_{e}<$ $v_{1}<\frac{\gamma}{\beta}$. Then $F_{\lambda}\left(v_{0}\right)=0$ and

$$
\begin{aligned}
& F_{\lambda}(y)<\frac{\beta}{\gamma} y, y \in\left(0, v_{e}\right), \\
& F_{\lambda}(y)>\frac{\beta}{\gamma} y, \quad y \in\left(v_{e}, \frac{\gamma}{\beta}\right) .
\end{aligned}
$$

Next we use the phase plane analysis for the following o.d.e. system which is equivalent to the previous second-order ordinary differential equation (65)

$$
v^{\prime}=w, \quad w^{\prime}=\Phi(v),
$$

with the "energy" $E$ and the "potential" $\mathcal{U}$ given by

$$
E(v, w)=\frac{1}{2} w^{2}+\mathcal{U}(v), \quad \mathcal{U}(v)=-\int_{v_{e}}^{v} \Phi_{\lambda}(y) d y .
$$

We denote by $x \mapsto \varphi\left(x ; \tilde{v}_{0}, \tilde{w}_{0}\right)=\left(\varphi^{1}\left(x ; \tilde{v}_{0}, \tilde{w}_{0}\right), \varphi^{2}\left(x ; \tilde{v}_{0}, \tilde{w}_{0}\right)\right)$ the solution to $(67)$ starting from $\left(\tilde{v}_{0}, \tilde{w}_{0}\right)$ at $x=0$ and by $T\left(\tilde{v}_{0}, \tilde{w}_{0}\right)$ the corresponding trajectory. We restrict the analysis of the phase portrait of (67) to the strip $\mathcal{P}=\left\{(v, w): v \in\left[0, \frac{\gamma}{\beta}\right]\right\}$ which 
contains three equilibria $Q_{i}, i \in\{0,1,2\}$. Since $\Phi(v)>0$ for $v \in\left(0, v_{e}\right)$ and $\Phi(v)<0$ for $v \in\left(v_{e}, \frac{\gamma}{\beta}\right)$, we realize that $\mathcal{U}$ has a local minimum at $v=v_{e}$ and $\mathcal{U}\left(v_{e}\right)=0$. It is easy to see that all the solutions are periodic. Trajectories contained in the strip $\mathcal{P}_{\omega}=\left\{(v, w): v \in\left(v_{0}, v_{1}\right)\right\}$ have period $\frac{2 \pi}{\omega}$.

Suppose now that $\tilde{v_{0}} \in\left(0, v_{e}\right)$ then we set

$$
\begin{aligned}
& v(x):=\varphi^{1}\left(x ; \tilde{v}_{0}, 0\right) \quad \text { for } x \in I, \\
& u(x):=f_{\lambda}(v(x)) \quad \text { for } x \in I,
\end{aligned}
$$

where the domain $I$ will be specified later in each case. Notice that $\tilde{w}_{0}=0$ ensures that the boundary condition (65) is satisfied.

Properties of solutions defined by (69)-(70) depend on the values of $\lambda$ and on the number of intersection points of trajectory $T\left(\tilde{v}_{0}, 0\right)$ with lines $l_{1}=\left\{(v, w): v=v_{0}\right\}$ and $l_{1}=\left\{(v, w): v=v_{0}\right\}$.

If $\lambda=\frac{\gamma}{2 \beta}$ then $\mathcal{U}(0)=\mathcal{U}\left(\frac{\gamma}{\beta}\right)$, and there is a heteroclinic trajectory that links equilibria $Q_{0}$ and $Q_{2}$. Applying Theorem 5 we find a family of stationary solutions $(u, v)$ that belong to $K$

$$
v=A \cos \omega x+\bar{v}, \quad x \in[0,2 \pi / \omega] .
$$

Then by (61) we obtain $A \leq \min \left\{A_{0}, A_{1}\right\}$ where

$$
A_{0}=\frac{\beta \bar{v}}{\gamma \chi_{0}}, \text { and } A_{1}=\frac{\gamma-\beta \bar{v}}{\gamma \chi_{0}}
$$

and $A_{0}\left(A_{1}\right)$ corresponds to $v_{0}$ (resp. $v_{1}$ ). Suppose now that $A_{0} \neq A_{1}$ i.e $\gamma \neq 2 \beta$. Then, it follows that $u$ never reaches 1 if $\bar{u}<1 / 2$ since then equivalently $\bar{v}<\frac{\gamma}{2 \beta}$ and $A_{0}<A_{1}$, which proves the last statement of the theorem. On the contrary, if $\bar{u}>1 / 2$ then $A_{0}>A_{1}$ and $u(x)=1$ for $x$ such that $|\cos \omega x|=1$. Since $\mathcal{U}\left(v_{0}\right)=\mathcal{U}\left(v_{1}\right)$ in this case taking $\tilde{v}_{0}=v_{0}$ or $\tilde{v}_{0}=v_{1}$ we obtain a solution to $(65)$ which belongs to $L^{\prime}$. If $\tilde{v}_{0} \in\left(0, v_{0}\right)$ then there is a solution in $L$.

If $\lambda<\frac{\gamma}{2 \beta}$ then $\mathcal{U}(0)<\mathcal{U}\left(\frac{\gamma}{\beta}\right)$ and there is a homoclinic trajectory emerging from $Q_{0}$ which surrounds point $Q_{1}$. In this case we have also $\mathcal{U}\left(v_{0}\right)<\mathcal{U}\left(v_{1}\right)<\mathcal{U}\left(\frac{\gamma}{\beta}\right)$. Therefore, given $\tilde{v}_{0} \in\left(0, v_{0}\right)$ the solution $x \mapsto \varphi\left(x ; \tilde{v}_{0}, 0\right)$ is determined and it defines the solution to (65) by means of (69)-(70). Such a solution belongs to the class $K_{0}$. Notice that in this case $\left[0, \frac{2 \pi}{\omega}\right] \subset I$. If $\tilde{v}_{0}=v_{0}$ then the corresponding solution (65) belongs to $K_{0}^{\prime}$ and in the case $\tilde{v}_{0} \in\left(v_{0}, v_{e}\right)$ the solution belongs to $K$ and then $\left[0, \frac{2 \pi}{\omega}\right]=I$. The level set corresponding to $\mathcal{U}\left(v_{0}\right)$ does not intersect line $l_{1}$ and therefore $u \neq 1$ provided $u \neq 0$.

If $\lambda>\frac{\gamma}{2 \beta}$ then $\mathcal{U}(0)>\mathcal{U}\left(\frac{\gamma}{\beta}\right)$, and there is a homoclinic orbit emerging from $Q_{2}$ which surrounds point $Q_{1}$. Moreover $\mathcal{U}\left(v_{0}\right)>\mathcal{U}\left(v_{1}\right)>\mathcal{U}\left(\frac{\gamma}{\beta}\right)$, and the third statement of the theorem follows by similar arguments to that in the previous case. It is then straightforward to check that the couple $(u, v)$ thus constructed is a stationary solution to (1)-(3) in the sense of Definition 4 and that $v \in \mathcal{C}^{2}([0, l])$ and $u \in \mathcal{C}([0, l])$. These two facts actually imply that $\mathcal{D}(u) \in \mathcal{C}^{1}([0, l])$. This completes the proof.

REMARK 9. A full characterization of the set of stationary states would be rather complicated since any function defined on $[0, l]$ which is a solution indicated in Theorem 8 restricted to $[0, l / 2]$ is also a stationary solution. Moreover, due to symmetry one can 
continue a solution defined on $[0, l]$ to a function defined on $[0,2 k l]$, where $k$ is a positive integer which is also a solution. Moreover merging of functions which preserves the regularity also produces new solutions on sufficiently large intervals.

\section{References}

[1] H. Amann, Nonhomogeneous linear and quasilinear elliptic and parabolic boundary value problems, in: Function Spaces, Differential Operators and Nonlinear Analysis, H. Triebel and H. J. Schmeisser (eds.), Teubner-Texte Math. 133, Teubner, Stuttgart, 1993, 9-126.

[2] T. Hillen and K. Painter, Global existence for a parabolic chemotaxis model with prevention of overcrowding, Adv. Appl. Math. 26 (2001), 280-301.

[3] H. M. Byrne and M. R. Owen A new interpretation of the Keller-Segel model based on multiphase modelling, J. Math. Biol. 49 (2004), 604-626.

[4] F. A. C. C. Chalub and J. F. Rodrigues, Kinetic models for chemotaxis with threshold, preprint, 2004.

[5] D. Horstmann, From 1970 until present: the Keller-Segel model in chemotaxis and its consequences. I, Jahresber. Deutsch. Math.-Verein. 105 (2003) 103-165.

[6] E. Keller and L. Segel, Initiation of slime mold aggregation viewed as an instability, J. Theor. Biology 26 (1970), 399-415.

[7] R. Kowalczyk, A. Gamba and L. Preciosi, On the stability of homogeneous solutions to some aggregation models, Discrete Contin. Dynam. Systems Series B 4 (2004), 204-220.

[8] Ph. Laurençot and D. Wrzosek, A chemotaxis model with threshold density and degenerate diffusion, in: Progress in Nonlinear Differential Equations and Their Applications 64, Birkhäuser, 2005, 273-290.

[9] J.-L. Lions, Quelques méthodes de résolution des problèmes aux limites non linéaires, Dunod, Paris, 1969.

[10] K. Painter and T. Hillen, Volume-filling and quorum-sensing in models for chemosensitive movement, Canadian Appl. Math. Q. 10 (2002), 501-543.

[11] C. S. Patlak, Random walk with persistence and external bias, Bull. Math. Biol. Biophys. 15 (1953), 311-338.

[12] J. Simon, Compact sets in the space $L^{p}(0, T ; B)$, Ann. Mat. Pura Appl. 146 (1987), 65-96.

[13] D. Wrzosek, Global attractor for a chemotaxis model with prevention of overcrowding, Nonlinear Anal. 59 (2004), 1293-1310.

[14] D. Wrzosek, Long time behaviour of solutions to a chemotaxis model with volume filling effect, Proc. Roy. Soc. Edinburgh 136A (2006), 431-444. 